\title{
Electrodynamic Characteristics of Multilayered System of Plane Screens with a Slot
}

\author{
M. E. Kaliberda, L. M. Lytvynenko' ${ }^{1}$, and S. A. Pogarsky \\ V. Karazin National University of Kharkiv, \\ 4, Svoboda Sq., Kharkiv, 61077, Ukraine \\ E-mail: Sergey.A.Pogarsky@univer.kharkov.ua \\ ${ }^{1}$ Institute of Radio Astronomy, National Academy of Sciences of Ukraine \\ 4, Chervonopraporna St., Kharkiv, 61002, Ukraine
}

Received March 28, 2011

The diffraction by a finite and semi-infinite system of plane screens with a slot is considered. The problem is solved with the operator approach. The possibility of screens shift in the plane of their displacement is taken into consideration.

Key words: operator equations, transmission and reflection operators, semi-infinite structure

\section{Introduction}

Multilayered strip structures are widely used in a number of applications, for example, in the creation of metamaterials, antennas, selective devices, etc. [1-3].

The practical usage of such structures requires the knowledge of fundamental solution of electromagnetic wave diffraction (in the general case, with an arbitrary space-time spectrum) by multilayered structures. Such problems belong to classical ones of electrodynamics $[4,5]$.

Periodic structures with strip conductors have become an integral part of a number of functional elements long ago, and in the first place, of antennas structures. For their synthesis the models, which allow to describe the properties of bounded periodic structures where fields have a continuous space spectrum, are essential. Thereupon, the study of characteristics of a semi-infinite and bounded system of plane screens with a slot is of great interest since the model where the field is represented as a wave beam, even two-dimensional, is practically adequate to real models. In this paper, an approach which describes the electrodynamic properties of a system of plane screens with a slot is proposed. This approach is one of the forms of the so-called semi-inversion method of the diffraction operator [6-8]. We may demonstrate the application of such an approach in determining the scattering operators of a finite-element and semi-infinite system of screens with a slot.

\section{Finite-Element System}

Let us place in free space a screen with a slot in the $z=0$ plane so that the origin of coordinates be placed in the middle of the slot. The slot width is $2 d$. In the $z=-n h$ plane let us place the $(n+1)$-th screen with a slot, where $n=1,2, \ldots M-1$, so that the $y$-coordinates of slot centers would differ for the neighboring screens by the $\Delta$-value. The coordinate system and structure geometry are shown in Fig. 1. Time dependence of electromagnetic field expressed as an exponential function of a negative imaginary power being proportional to circular frequency and time is implied everywhere in the following.

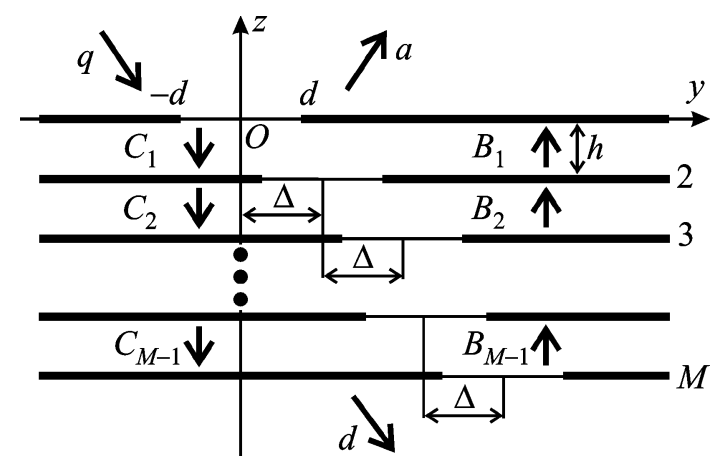

Fig. 1. Finite-element structure 
For the $E$-polarization case, consider the electric field $E_{x}$ component. Let us represent the incident field from the half-space $z>0$ as

$$
E_{x}^{i}(y, z)=\int_{-\infty}^{\infty} q(\xi) \exp (i k \xi y-i k \gamma(\xi) z) \mathrm{d} \xi
$$

where $\gamma(\xi)=\sqrt{1-\xi^{2}}$, Re $\gamma \geq 0, \operatorname{Im} \gamma \geq 0$. We suppose that the transmission $t$ and reflection $r$ operators of a single screen with a slot are known. Their action on arbitrary function $g(\zeta)$ is described by expressions

$$
\begin{aligned}
& (\operatorname{tg})(\xi)=\int_{-\infty}^{\infty} t(\xi, \zeta) g(\zeta) \mathrm{d} \zeta, \\
& (r g)(\xi)=\int_{-\infty}^{\infty} r(\xi, \zeta) g(\zeta) \mathrm{d} \zeta,
\end{aligned}
$$

and

$$
r(\xi, \zeta)=t(\xi, \zeta)-\delta(\xi-\zeta)
$$

Following [7, 8], we denote Fourier amplitudes of the reflected, transmitted fields and the field between screens as $a(\xi), d(\xi), C_{n}(\xi)$ and $B_{n}(\xi)$, respectively, where $n=1, \ldots, M-1$. Using expression (1), these amplitudes in the operator notations are related as follows

$$
\begin{aligned}
& a=t q-q+t^{-} B_{1}, \\
& C_{1}=t q+t B_{1}-B_{1}, \\
& C_{n}=\operatorname{tes}^{+} C_{n-1}+\operatorname{tes}^{-} B_{n}-e s^{-} B_{n}, \quad n=2, \ldots, M-1,
\end{aligned}
$$

$$
B_{n}=\text { tes }^{+} C_{n}-e s^{+} C_{n}+\text { tes }^{-} B_{n+1}, \quad n=1, \ldots, M-2,
$$

$$
\begin{aligned}
& B_{M-1}=\operatorname{tes}^{+} C_{M-1}-\operatorname{es}^{+} C_{M-1}, \\
& d=\operatorname{tes}^{+} C_{M-1},
\end{aligned}
$$

where operator $e$ determines the amplitude variation of the field that occurs when the coordinate system is shifted by the distance $h$ along the $z$-axis toward field propagation. Operators $s^{ \pm}$determine the field amplitude variation that occurs when the coordinate system is shifted by the $\Delta$-distance either in positive or negative directions along the $y$-axis. The system of equations (2)-(7) may be considered as that of integral equations.

In the case when the plane waveguide eigenwaves propagation between layers is possible, parameter $k h>\pi$, functions $C_{n}(\xi)$ and $B_{n}(\xi)$, where $n=1, \ldots, M-1$, have singularities in the points $\beta_{p}=\operatorname{sgn}(p) \sqrt{1-\left(\frac{\pi p}{k h}\right)^{2}}$, where $p=-N, \ldots, N$, $p \neq 0, \quad N=\left[\frac{k h}{\pi}\right]$, which correspond to propagation constants of waveguide eigenwaves. Here notation [.] denotes integer part of a number. First, we assume that the excitation frequency does not coincide with the cutoff frequency of plane waveguide eigenwaves, i. e. parameter $k h \neq \pi N$. Then these singularities are the poles of first order. Now introduce functions $C_{n}^{1}(\xi)$ and $B_{n}^{1}(\xi)$ as follows

$$
\begin{aligned}
& C_{n}(\xi)=\frac{C_{n}^{1}(\xi)}{1-\exp (2 i k h \gamma(\xi))}, \\
& B_{n}(\xi)=\frac{B_{n}^{1}(\xi)}{1-\exp (2 i k h \gamma(\xi))} .
\end{aligned}
$$

The poles excluding (regularization procedure) yields

$$
\begin{aligned}
& a=t q-q+t^{-}{ }^{-} G B_{1}^{1}, \\
& \left(I-e^{2}\right)^{-1} C_{1}^{1}=t q+t G B_{1}^{1}-\left(I-e^{2}\right)^{-1} B_{1}^{1}, \\
& \left(I-e^{2}\right)^{-1} C_{n}^{1}=t_{e s}^{+} G C_{n-1}^{1}+\operatorname{tes}^{-} G B_{n}^{1} \\
& -e^{-}\left(I-e^{2}\right)^{-1} B_{n}^{1}, \quad n=2, \ldots, M-1,
\end{aligned}
$$

$$
\begin{aligned}
& \left(I-e^{2}\right)^{-1} B_{n}^{1}=\operatorname{tes}^{+} G C_{n}^{1}-e^{+}\left(I-e^{2}\right)^{-1} C_{n}^{1} \\
& +\operatorname{tes}^{-} G B_{n+1}^{1}, \quad n=1, \ldots, M-2,
\end{aligned}
$$

$$
\left(I-e^{2}\right)^{-1} B_{M-1}^{1}=\operatorname{tes}^{+} G C_{M-1}^{1}-e s^{+}\left(I-e^{2}\right)^{-1} C_{M-1}^{1},
$$

$d=\operatorname{tes}^{+} G C_{M-1}^{1}$, 
( $I$ is the unity operator).

The integrands in these equations do not have singularities and operator $G$ acts on arbitrary function $q(\zeta)$ as follows

$$
(G q)(\xi)=\chi(\xi) \frac{q(\xi)}{1-\exp (2 i k h \gamma(\xi))}
$$

$+\left(\bar{\chi}(\xi) \frac{q(\xi)}{1-\exp (2 i k h \gamma(\xi))}-\sum_{\substack{p=-N \\ p \neq 0}}^{N} \delta\left(\xi-\beta_{p}\right)\right.$

$\left.\times \int_{M_{1}}^{M_{2}} \frac{\sigma_{p} q\left(\beta_{p}\right)}{\zeta-\beta_{p}} d \zeta\right)+\sum_{\substack{p=-N \\ p \neq 0}}^{N} \delta\left(\xi-\beta_{p}\right) \sigma_{p} q\left(\beta_{p}\right)$

$\times\left\{\ln \left(\frac{M_{2}-\beta_{p}}{\beta_{p}-M_{1}}\right)+\pi i \operatorname{sgn}(p)\right\}$,

where $\left[M_{1} ; M_{2}\right] \supset[-1 ; 1], \quad \sigma_{p}=-\frac{i \pi|p|}{2 k^{2} h^{2} \beta_{p}}$,

$$
\begin{aligned}
& \chi(\xi)= \begin{cases}1, & \xi \notin\left[M_{1}, M_{2}\right], \\
0 & \xi \in\left(M_{1}, M_{2}\right),\end{cases} \\
& \bar{\chi}(\xi)= \begin{cases}0, & \xi \notin\left[M_{1}, M_{2}\right], \\
1 & \xi \in\left(M_{1}, M_{2}\right) .\end{cases}
\end{aligned}
$$

In the case when the excitation frequency coincides with the cutoff frequency of one of the waveguide waves, i. e. $k h=\pi N$, then in expression (8) $p \neq \pm N$ and integral in the neighborhood of points $\beta_{ \pm N}$ should be considered as Cauchy principal value [9].

\section{Semi-Infinite System}

Let us use the same notations in this section as in the case of a finite-element system with the only difference that in the case of a semiinfinite structure we should assume $M=\infty$. The structure geometry and coordinate system are shown in Fig. 2. The Fourier amplitudes of the reflected field and these between screens are related as follows

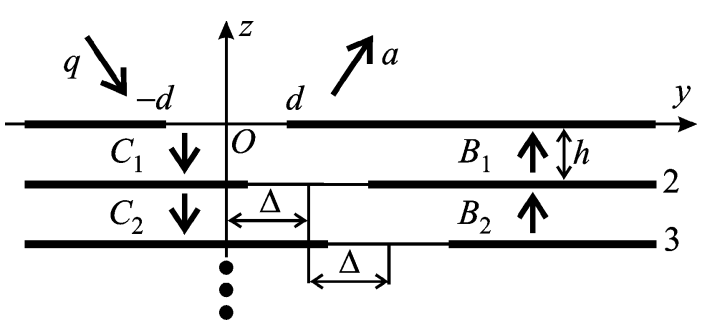

Fig. 2. Semi-infinite structure

$a=\tilde{R} q$,

$a=t q-q+t^{-} s^{-}$,

$C_{1}=t q+t^{-}{ }^{-} B_{1}-e s^{-} B_{1}$,

$B_{n}=\tilde{R}_{e s}{ }^{+} C_{n}, \quad n=1,2, \ldots$,

$C_{n}=$ tes $^{+} C_{n-1}+$ tes $^{-} B_{n}-e^{-} B_{n}, \quad n=2,3, \ldots$,

where operator $\tilde{R}$ is the sought for operator of a semi-infinite structure. Now introduce notations

$R(\xi, \zeta)=\tilde{R}(\xi, \zeta)+\delta(\xi-\zeta), \quad C_{1}=C\left(I-e^{2}\right)^{-1}$

The transformations of equations (9)-(12) and regularization procedure, with the use of relation (1), yield

$R q=t q+t^{-} s^{-} \operatorname{Res}^{+} G C-t e^{2} G C$

$C=\left(I+e s^{-} \operatorname{Res}^{+} G\right)^{-1} R q$.

After substitution $C$ from equation (15) into equation (14) we obtain operator equation to determine the operator $R$,

$$
\begin{aligned}
& R=t+\operatorname{tes}^{-} \operatorname{Res}^{+} G\left(I+\operatorname{es}^{-} \operatorname{Res}^{+} G\right)^{-1} R \\
& -t e^{2} G\left(I+\operatorname{es}^{-} \operatorname{Res}^{+} G\right)^{-1} R .
\end{aligned}
$$


The amplitudes of field between the $n$-th and the $(n+1)$-th layers may be obtained from equations (12) and (13).

For the $H$-polarization case, instead of relation (1) use the following relation

$r(\xi, \zeta)=t(\xi, \zeta)+\delta(\xi-\zeta)$

and introduce the functions

$$
\begin{aligned}
& C_{n}(\xi)=\frac{C_{n}^{1}(\xi)}{\gamma(\xi)\{1-\exp (2 i k h \gamma(\xi))\}}, \\
& B_{n}(\xi)=\frac{B_{n}^{1}(\xi)}{\gamma(\xi)\{1-\exp (2 i k h \gamma(\xi))\}} .
\end{aligned}
$$

Relation (8) should be rewritten in the form

$$
\begin{aligned}
& (G q)(\xi)=\chi(\xi) \frac{q(\xi)}{\gamma(\xi)\{1-\exp (2 i k h \gamma(\xi))\}} \\
& +\left(\bar{\chi}(\xi) \frac{q(\xi)}{\gamma(\xi)\{1-\exp (2 i k h \gamma(\xi))\}}\right. \\
& -\sum_{p=-N}^{N} \delta\left(\xi-\beta_{p}\right) \int_{M_{1}}^{M_{2}} \frac{\sigma_{p} q\left(\beta_{p}\right)}{\zeta \neq \beta_{p}} \mathrm{~d} \zeta \\
& \left.-\delta(\xi+1) \int_{M_{1}}^{M_{2}} \frac{\sigma_{-1}^{1} q(-1)}{\zeta+1} \mathrm{~d} \zeta-\delta(\xi-1) \int_{M_{1}}^{M_{2}} \frac{\sigma_{1}^{1} q(1)}{\zeta-1} \mathrm{~d} \zeta\right) \\
& +\sum_{\substack{p=-N \\
p \neq 0}}^{N} \delta\left(\xi-\beta_{p}\right) \sigma_{p} q\left(\beta_{p}\right) \\
& \times\left\{\ln \left(\frac{M_{2}-\beta_{p}}{\beta_{p}-M_{1}}\right)+\pi i \operatorname{sgn}(p)\right\} \\
& +\delta(\xi+1) \sigma_{-1}^{1} q(-1)\left\{\ln \left(\frac{M_{2}+1}{-1-M_{1}}\right)-\pi i\right\} \\
& +\delta(\xi-1) \sigma_{1}^{1} q(1)\left\{\ln \left(\frac{M_{2}-1}{1-M_{1}}\right)+\pi i\right\} \\
& \\
& +(\xi)
\end{aligned}
$$

where $\sigma_{p}=-\frac{i}{2 k h \beta_{p}}, \quad \sigma_{ \pm 1}^{1}=\mp \frac{i}{4 k h}$.

\section{Numerical results}

Fig. 3 shows the dependences of transmission $\tau$ and reflection $\rho$ coefficients of a double screen with a slot as functions of screen shift $\Delta$ along the $y$-axis for the cases of normal incidence, $\varphi_{0}=90^{\circ}$, and of an angle of incidence $\varphi_{0}=30^{\circ}$ (see insert of Fig. 3). The distance between screens is $h=5 / k$. With such value of parameter $k h$, the $T E_{01}$-wave can propagate between screens. The dependences of excitation factors of right $t^{+}$and left $t^{-}$waveguides are shown in Fig. 4 as functions of parameter $\Delta$. The transmission, reflection and waveguide excitation factors are calculated from the formulas

$$
\rho=\frac{\pi}{k d \sin \varphi_{0}} \int_{-1}^{1}\left|a(\xi)+\delta\left(\xi-\cos \varphi_{0}\right)\right|^{2} \sqrt{1-\xi^{2}} \mathrm{~d} \xi,
$$

$$
\begin{aligned}
& \tau=\frac{\pi}{k d \sin \varphi_{0}} \int_{-1}^{1}|d(\xi)|^{2} \sqrt{1-\xi^{2}} \mathrm{~d} \xi, \\
& t^{ \pm}=\frac{\pi^{2}}{k d k h \sin \varphi_{0}} \\
& \times \sum_{p= \pm 1}^{ \pm N}\left|C\left(\beta_{p}\right)\left\{1-\exp \left(2 i k h \gamma\left(\beta_{p}\right)\right)\right\}\right|^{2} \frac{1-\beta_{p}^{2}}{\left|\beta_{p}\right|} .
\end{aligned}
$$

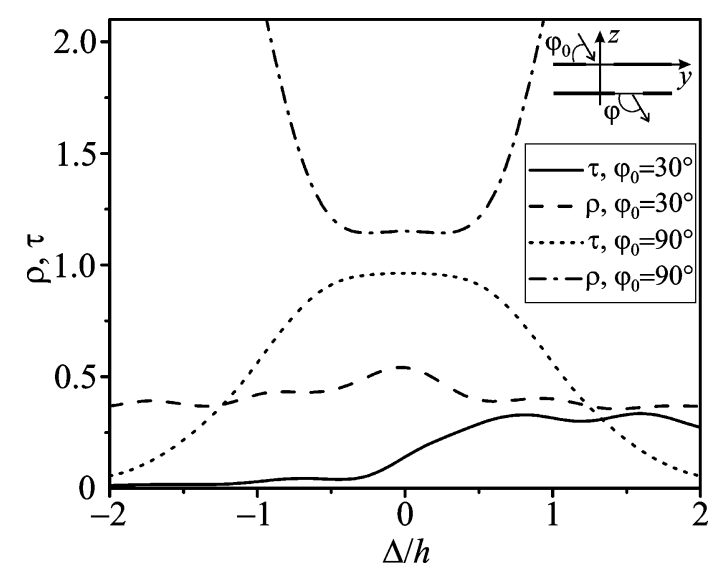

Fig. 3. Dependences of transmission (solid line) and reflection (dashed line) coefficients for the case of an angle of incidence $\varphi_{0}=30^{\circ}$, and transmission (dotted line) and reflection (dashed-dotted line) coefficients for the case of normal incidence, $\varphi_{0}=90^{\circ}, v s . \Delta$ of a double screen with a slot. The structure parameters are $k h=5, d / h=1$ 


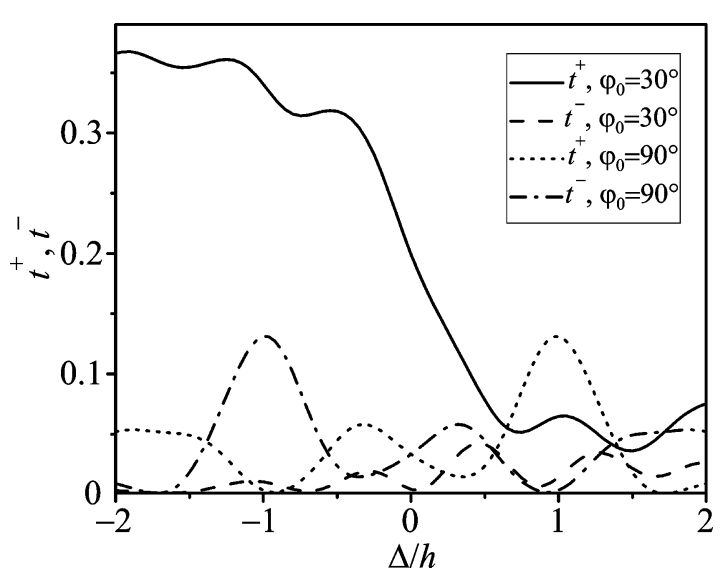

Fig. 4. Dependences of right (solid line), left (dashed line) waveguide excitation factors for the case of an angle of incidence $\varphi_{0}=30^{\circ}$, and right (dotted line), left (dashed-dotted line) waveguide excitation factors for the case of normal incidence, $\varphi_{0}=90^{\circ}$, vs. $\Delta$ of a double screen with a slot. The structure parameters are $k h=5, d / h=1$

In the case of normal incidence, the curves of transmission and reflection coefficients are symmetrical with respect to line $\Delta / h=0$, and the curve of right-waveguide excitation factor equals to the curve of left-waveguide excitation factor and is reflected symmetrically over the same line. In the case of angle of incidence $\varphi_{0}=30^{\circ}$, when $\Delta / h<0$, the transmitted field is practically absent. In this case, virtually all energy of scattered field is consumed by plane waveguide excitation and the values of right-waveguide excitation factor are significantly greater than those of left-waveguide excitation factor. When the second screen center approaches the $y=0$ plane, the incident field passes through the slots into half-space $z<-h$, and the transmission coefficient increases. The module of normalized directional patterns of transmitted field is shown in Fig. 5. The directional pattern of transmitted field is calculated from the formula

$$
D^{1}(\varphi)=\sqrt{2 \pi / k} d(-\cos (\varphi)) \sin (\varphi) e^{-i \pi / 4}, \quad \varphi \in[0, \pi] .
$$

The value of parameter $\Delta$ is chosen so that the reflection coefficient in the case of normal incidence coincide with the reflection coefficient for the case of an angle of incidence $\varphi_{0}=30^{\circ}$. As the figure shows, when we change the incidence angle by $60^{\circ}$ the main lobe rotates approximately by $10^{\circ}$.

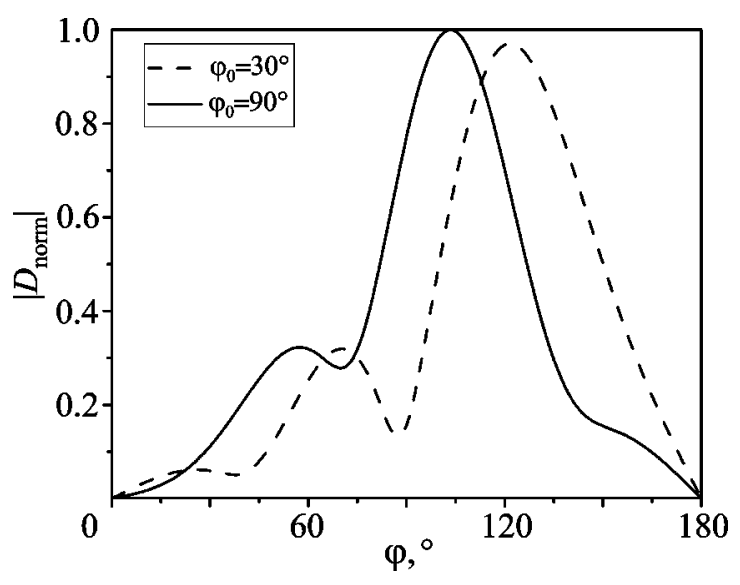

Fig. 5. Module of normalized directional patterns of transmitted field of a double screen with a slot for the cases $\varphi_{0}=30^{\circ}$ (dashed curve) and $\varphi_{0}=90^{\circ}$ (solid curve)

Fig. 6 shows the dependences of transmission $\tau$ and reflection $\rho$ coefficients of a structure of six layers and reflection coefficient $\rho_{\infty}$ of a semi-infinite structure as functions of parameter $\Delta$. As in the case of a double screen, when parameter $|\Delta|$ is decreased, the reflection coefficient is also decreased while the transmission coefficient increased. But in the six-element structure case, the quasi-passband becomes narrower than in the two-element structure case. The reflection coefficient of a finite-element structure approaches the reflection coefficient

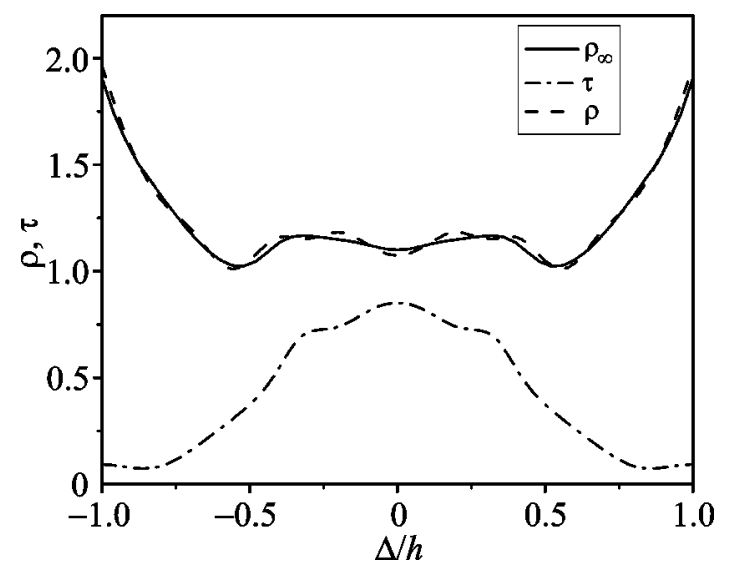

Fig. 6. Dependences of reflection coefficient of a semiinfinite structure (solid line), and transmission (dashed-dotted line) and reflection (dashed line) coefficients of a six-element structure vs. $\Delta, \varphi_{0}=90^{\circ}$. The structure parameters are $k h=5, d / h=1$ 


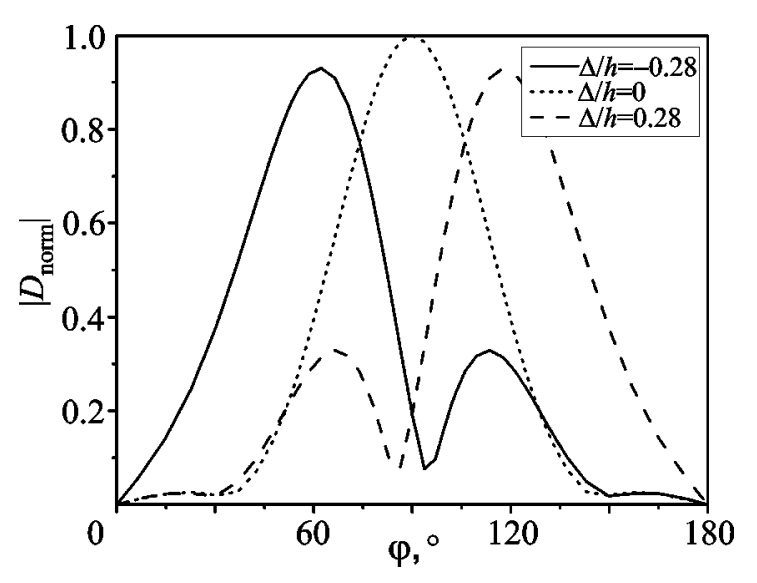

Fig. 7. Module of normalized directional patterns of transmitted field of a six-element structure for $\Delta / h=-0.28$ (solid line), $\Delta / h=0$ (dotted line) and $\Delta / h=0.28$ (dashed line), $\varphi_{0}=90^{\circ}$. The structure parameters are $k h=5, d / h=1$

of a semi-infinite structure when the number of screens is increased.

Fig. 7 shows the module of normalized directional patterns of transmitted field for a structure of six layers. Normalization is performed by the maximum of module of the directional pattern when $\Delta=0$. Variation of parameter $\Delta$ leads to rotation of the antenna pattern main lobe. In our case, one can observe the rotation by the angle of $27^{\circ}$ with respect to normal, and simultaneous decreasing of main lobe level by no more than $7 \%$. The further increasing of parameter $\Delta \mid$ leads to significant decreasing of lobe level. This follows from the dependence of transmission coefficient of this structure as a function of parameter $\Delta$.

\section{Conclusions}

The diffraction by the finite-element and semiinfinite systems of plane screens with a slot is solved. The presented approach may be applied to solve the synthesis problems of antenna devices with controlled antenna patterns, excitation of open periodic structures, and creation of metamaterials.

\section{References}

1. L. M. Lytvynenko and S. L. Prosvirnin, "Wave reflection by a periodic layered metamaterial", The European Physical Journal Applied Physics, vol. 46, 32608-(p1-p6), 2009.

2. X. H. Wu, A. A. Kishk, and A. W. Glisson, "A Transmission Line Method to Compute the Far-Field Radia- tion of Arbitrary Hertzian Dipoles in a Multilayer Structure Embedded With PEC Strip Interfaces", IEEE Trans. Antennas Propag., vol. 55, no. 11, pp. 3191-3198, 2007.

3. A. K. Bhattacharyya, "Analysis of Multilayer Infinite Periodic Array Structures with Different Periodicities and Axes Orientations", IEEE Trans. Antennas Propag., vol. 48, no. 3, pp. 357-369, 2000.

4. B. Noble, Methods Based on the Wiener-Hopf Technique for the Solution of Partial Differential Equations, Oxford: Pergamon, 1958, $263 \mathrm{p}$.

5. T. B. A. Senior, "Diffraction by a semi-infinite metallic sheet", Proc. R. Soc. A., vol. 213, No. 1115. pp. 436-458, 1952.

6. K. Schwarzschild, "Die Beugung und Polarisation des Lichts durch einen Spalt. I", Math. Ann., vol. 5, pp. 177-247, 1902.

7. L. M. Lytvynenko and S. L. Prosvirnin. Spectral scattering operators in diffraction by plane screens, Kyiv: Naukova Dumka, 1984, 239 p. (in Russian).

8. M. E. Kaliberda, L. M. Lytvynenko, and S. A. Pogarskii, "Operator Method in the Analysis of Electromagnetic Wave Diffraction by Planar Screens", J. Comm. Tech. Electron., vol. 54, no. 9, pp. 975-981, 2009.

9. L. M. Lytvynenko and S. L. Prosvirnin, "Transverse slot in a plane waveguide", Radiotekhnika i Electronika, vol. 22, no. 7, pp. 1321-1326, 1977, (in Russian).

\section{Электродинамические характеристики многослойной системы плоских экранов со щелью}

\section{М. Е. Калиберда, Л. Н. Литвиненко, С. А. Погарский}

Рассмотрена здача дифракции на конечноэлементной и полубесконечной системе плоских экранов со щелью. Решение найдено при помощи операторного метода. Учтена возможность смещения экранов в плоскости их расположения.

\section{Електродинамічні характеристики багатошарової системи плоских екранів зі щілиною}

\section{М. Є. Каліберда, Л. М. Литвиненко, С. О. Погарський}

Розглянуто задачу дифракції на скінченноелементній та напівнескінченній системі плоских екранів зі щілиною. Розв'язок знайдено за допомогою операторного методу. Враховано можливість зміщення екранів у площині їх розташування. 